\title{
Osteoprotegerin and its cognate ligand: a new paradigm of osteoclastogenesis
}

\author{
Lorenz C Hofbauer and Armin E Heufelder ${ }^{1}$ \\ Endocrine Research Unit, Mayo Clinic, Rochester, Minnesota, USA and ${ }^{1}$ Division of Endocrinology, Medizinische Klinik, Klinikum Innenstadt, \\ Ludwig-Maximilians-Universität, Munich, Germany \\ (Correspondence should be addressed to L C Hofbauer, Endocrine Research Unit, Joseph 5-164, Mayo Clinic, Rochester, Minnesota 55905, USA)
}

Osteoprotegerin (OPG) has recently been identified as a new member of the tumor necrosis factor (TNF)receptor (TNF-R) superfamily (1-3). It is a glycoprotein with seven structural domains, including four TNF-R motifs at the N-terminus (domains 1-4), two death domain homologous regions (domains 5 and 6) which mediate cytotoxicity, and a C-terminus (domain 7) which contains a cysteine residue for homodimer formation (4). In contrast with other known members of the TNF-R superfamily, OPG (or osteoclastogenesis inhibitory factor, OCIF) lacks transmembrane domains, suggesting that it acts as a secreted cytokine receptor (1-4). Enthusiasm for this new cytokine receptor emerged from basic scientists and clinicians alike after it was shown that, by suppressing osteoclastogenesis, OPG is a potent inhibitor of bone resorption $(1,2,5,6)$. Moreover, overexpression of OPG in transgenic mice resulted in osteopetrosis (generalized increase in bone mass) (1) and prevented bone loss following ovariectomy $(1,2)$. The potent antiresorptive effects of OPG nurtured the hope that deciphering its mode of action would provide detailed insights into osteoclastogenesis, an integral part of the pathogenesis of metabolic bone diseases. However, while the effect of OPG was impressive and comparable with the most potent class of antiresorptive drugs, the bisphosphonates, the cognate ligand for OPG had remained unidentified, rendering OPG another orphan receptor and leaving a major piece of the puzzle of osteoclastogenesis missing.

One year later, the cognate ligand for OPG (OPGL) has been identified independently by two groups $(7,8)$. After screening of OPG-binding cell surface proteins with an OPG immunoprobe, OPGL was expression-cloned and the human OPGL cDNA was isolated from lymph node (7) and stromal cell (8) cDNA libraries. Human OPGL (or osteoclast differentiation factor, ODF) consists of 317 amino acids and belongs to the class of type II transmembrane proteins $(7,8)$. OPGL exists in a cellbound and a soluble C-terminal form, both of which are bound by OPG (7). It is highly expressed in lymphoid tissues (lymph node, thymus, spleen) and trabecular bone, and to a lesser extent in bone marrow as well as in some bone marrow stromal and osteosarcoma cell lines $(7,8)$. OPGL mRNA expression in the murine marrow stromal cell line, ST2, is upregulated by vitamin D.
Furthermore, vitamin D, interleukin-11, prostaglandin $\mathrm{E}_{2}$ and parathyroid hormone increased OPGL mRNA expression in primary mouse osteoblasts (8). Within fetal bone, OPGL is mainly expressed in the cartilaginous anlagen and in hypertrophic chondrocytes, while in adult bone its expression is most abundant in the growth plate and the metaphysis, all of which are areas of active bone remodeling during growth (7). OPGL binds to the cell surface of osteoclastic lineage cells, and, in the presence of the permissive factor colony-stimuating factor-1 (CSF-1), it induces osteoclastogenesis in vitro in the absence of stromal cells, glucocorticoids and vitamin $\mathrm{D}$, factors previously considered essential for osteoclastogenesis $(7,8)$. Importantly, OPGL stimulated bone resorption both in vitro $(7,8)$ and in vivo $(7)$. The latter (after subcutaneous injection of recombinant OPGL into normal mice) was associated with an increased size and nuclearity of osteoclasts, massive bone loss and profound rapid-onset hypercalcemia (7).

These studies unequivocally establish OPGL and OPG as a cybernetic couple that regulates bone mass by modulating osteoclastogenesis. OPGL appears to be the endogenous 'master' cytokine, which is the conditio sine qua non for normal osteoclast differentiation and activation, whereas OPG is a naturally occurring soluble receptor that counterbalances the effects of OPGL and preserves bone mass (Fig. 1). This concept is further supported by increased osteoclastogenesis and severe osteopenia in OPG knock-out mice (as cited in (7)). Sequence comparison of the $\mathrm{OPG}$ ligand $(7,8)$ has revealed that it is identical with two previously reported novel members of the TNF-R superfamily, TNF-related activation-induced cytokine (TRANCE) (9) and receptor activator of $N F-\kappa B$ ligand (RANKL) (10), which are essential for T-cell and dendritic cell activation. TRANCE was identified as an immediate early gene that is upregulated by T-cell receptor stimulation. Its expression is restricted to lymphoid tissue (thymus, lymph nodes) and T-cells, and it induces activation of the c-jun N-terminal kinase in T-cells (9). RANKL was identified by screening a thymoma cell line which highly expressed RANK, its cognate receptor, and was found to be mainly expressed in lymph node, with lower expression in other tissues such as placenta, bone marrow and thyroid (10). Subsequently, RANKL was 


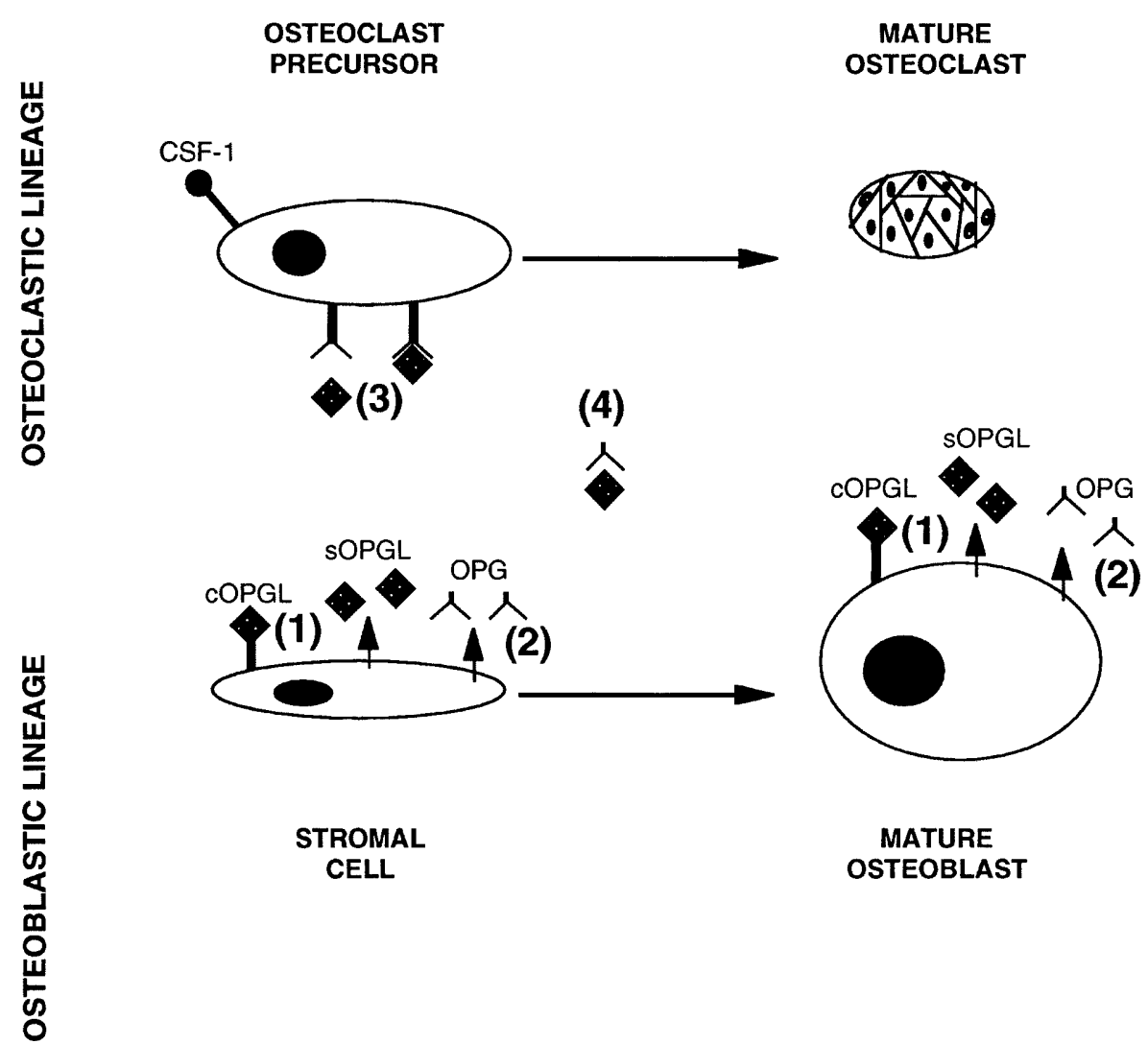

Figure 1 Mechanisms of OPG and OPGL in regulating osteoclastogenesis. (1) Stromal cells and mature osteoblasts express cell membrane-bound (COPGL) and soluble OPGL (sOPGL). (2) Stromal cells and mature osteoblasts secrete OPG, a soluble OPGL antagonist. (3) Both forms of OPGL bind to their cognate receptor located on osteoclast precursor cells, and, in the presence of permissive factors such as CSF-1 promote osteoclastogenesis. (4) OPG counteracts the effects described in (3) by competing for, and neutralizing OPGL.

found to specifically stimulate aggregation, cluster formation, and allostimulatory capacity of dendritic cells, which are surrogates of dendritic cell function to capture, process and present antigens to T-cells (10).

The discovery of OPG and its cognate ligand OPGL/ TRANCE/RANKL has created a new paradigm of osteoclastogenesis and has underlined the role of pleiotropic cytokines, as well as their cognate receptors and antagonists, in the microenvironment of bone marrow (11). Future studies are likely to demonstrate a further linkage between the hematopoietic and the immune system and bone metabolism, which all derive from common pluripotent stem cells residing within the confines of bone and its marrow.

\section{References}

1 Simonet WS, Lacey DL, Dunstan CR, Kelley M, Chang M-S, Lüthy R et al. Osteoprotegerin: a novel secreted protein involved in the regulation of bone density. Cell 199789 309-319.

2 Yasuda H, Shima N, Nakagawa N, Mochizuki S-I, Yano K, Fujise $\mathrm{N}$ et al. Identity of osteoclastogenesis inhibitory factor (OCIF) and osteoprotegerin (OPG): a mechanism by which OPG/
OCIF inhibits osteoclastogenesis in vitro. Endocrinology 199839 1329-1337.

3 Tan KB, Harrop J, Reddy M, Young P, Terrett J, Emery J et al. Characterization of a novel TNF-like ligand and recently described TNF ligand and TNF receptor superfamily genes and their constitutive and inducible expression in hematopoietic and nonhematopoietic cells. Gene 1997204 35-46.

4 Yamaguchi K, Kinosaki M, Goto M, Kobayashi F, Tsuda E, Morinaga $\mathrm{T}$ et al. Characterization of structural domains of human osteoclastogenesis inhibitory factor. Journal of Biological Chemistry $19982735117-5123$.

5 Tsuda E, Goto M, Mochizuki S-I, Yano K, Kobayashi F, Morinaga T et al. Isolation of a novel cytokine from human fibroblasts that specifically inhibits osteoclastogenesis. Biochemical and Biophysical Research Communications 1997234 137-142.

6 Miyamoto A, Kunisada T, Hemmi H, Yamane T, Yasuda H, Miyake K et al. Establishment and characterization of an immortal macrophage-like cell line inducible to differentiate to osteoclasts. Biochemical and Biophysical Research Communications 1998242 703-709.

7 Lacey DL, Timms E, Tan H-L, Kelley MJ, Dunstan CR, Burgess T et al. Osteoprotegerin (OPG) ligand is a cytokine that regulates osteoclast differentiation and activation. Cell 199893 165-176.

8 Yasuda H, Shima N, Nakagawa N, Yamaguchi K, Kinosaki M, Mochizuki S-I et al. Osteoclast differentiation factor is a ligand for osteoprotegerin/osteoclastogenesis-inhibitory factor and is identical to TRANCE/RANKL. Proceedings of the National Academy of Sciences of the USA 199895 3597-3602. 
9 Wong BR, Rho J, Arron J, Robinson E, Orlinick J, Chao M et al. TRANCE is a novel ligand of the tumor necrosis factor receptor family that activates c-jun N-terminal kinase in T cells. Journal of Biological Chemistry $199727225190-25194$.

10 Anderson MA, Maraskovsky E, Billingsley WL, Dougall WC, Tometsko ME, Roux ER et al. A homologue of the TNF receptor and its ligand enhance T-cell growth and dendritic-cell function. Nature 1997390 175-179.

11 Sharrock WJ, for the Organizers and Speakers. Bone and the hematopoietic and immune systems: a report of the proceedings of a scientific workshop. Journal of Bone and Mineral Research 1998 13 537-543. 